\title{
Percepción del estado situacional de la gestión administrativa para efectivizar la defensa del patrimonio cultural arqueológico en la región Lambayeque, año 2020
}

\section{Perception of the situational state of administrative management to make the defense of archaeological cultural heritage effective in the Lambayeque region, year 2020}

\author{
Antonio Chucchucan ${ }^{1}$ iD *
}

\section{RESUMEN}

El presente estudio tuvo como objetivo principal identificar el nivel de percepción del estado situacional de la gestión administrativa para efectivizar la defensa del patrimonio cultural arqueológico en la región Lambayeque, 2020. El tipo de diseño de investigación fue la no experimental y descriptivo propositivo. Para ello se planteó la implementación de estrategias de gestión. La investigación tuvo como muestra a 76 trabajadores del Ministerio de Cultura, el instrumento de recolección de datos fue la encuesta destinada a los trabajadores del Ministerio de Cultura, tales como el encargado de la defensa del Patrimonio Cultural de la Nación de Lambayeque, profesionales de arqueología, trabajadores de los museos de la región, los empleados de la unidad ejecutora y el director representante del Ministerio de Cultura. Los resultados indican que la gestión de la defensa de patrimonio cultural es inadecuada en un $100 \%$, ya que no existe el debido interés, ni el presupuesto para su gestión, por último, se llegó a validar la hipótesis de investigación en el cual la aplicación de estrategias de planificación, organización, dirección y el control, conlleva a efectivizar la defensa del patrimonio arqueológico de la región Lambayeque, 2020.

Palabras clave: Planificación, organización, valor de uso, valor simbólico.

\begin{abstract}
The main objective of the present study was to identify the level of perception of the situational state of administrative management in order to effectively defend the archaeological cultural heritage in the Lambayeque region, 2020. The type of research design was non-experimental and descriptive and propositional. For this purpose, the implementation of management strategies was proposed. The research sample consisted of 76 employees of the Ministry of Culture, the data collection instrument was a survey aimed at Ministry of Culture workers, such as the person in charge of the defence of the Cultural Heritage of the Nation of Lambayeque, archaeology professionals, museum workers in the region, employees of the executing unit and the representative director of the Ministry of Culture. The results indicate that the management of the defence of cultural heritage is inadequate in $100 \%$, since there is neither the due interest, nor the budget for its management, finally, we came to validate the research hypothesis in which the application of strategies of planning, organisation, direction and control, leads to make effective the defence of the archaeological heritage of the Lambayeque region, 2020.
\end{abstract}

Keywords: Planning, organization, use value, symbolic value.

DOI: https://doi.org/10.37787/pakamuros-unj.v9i3.216

Recibido: 22/03/2020. Aceptado: 23/09/2021

* Autor para correspondencia

1. Universidad Privada Cesar Vallejo -Chiclayo, Perú. Email: chbrionesa@ucvvirtual.edu.pe 


\section{INTRODUCCIÓN}

Mendoza (2011) en su investigación realizada en México manifiesta que el Patrimonio Cultural Arqueológico se encuentra en riesgo, pues están padeciendo de la urbanización descontrolada, ocasionando la transformación y el deterioro de centros culturales. Baeza (2015) indica que en Chile el evento llamado Dakar es visto negativamente desde una mirada cultural, siendo el principal problema la circulación de vehículos entre automóviles, camiones, cuadriciclos y motos quienes avanzan a gran velocidad por el desierto de Atacama, originando el deterioro del patrimonio cultural. En el contexto nacional, la integridad del patrimonio también se ve perjudicado, especialmente por la invasión de zonas arqueológicas.

Noreña (2013) en su artículo sobre valoración del patrimonio arqueológico en la ciudad de Trujillo en especial en la zona de Huanchaco, manifiesta que un grupo de personas invadieron un promedio de 300 metros de terreno de la zona arqueológica denominada Gramalote perteneciente a la cultura Chimú, los invasores pretendían presentar documentos falsos los cuales garantizaban que el área del terreno era de su propiedad.

Alva (2016) menciona que existen complejos arqueológicos que se encuentran en peligro de destrucción, uno de ellos es el complejo arqueológico de Mata Indio, ubicado en Zaña, el cual fue invadido por un grupo de personas armadas asegurando que era propiedad privada, cercando dicha zona a pesar que la Dirección Desconcentrada de Cultura de Lambayeque (DDC-Lambayeque) habría intervenido, los invasores están procediendo a repartirse los terrenos y a la venta de lotes para uso agrícola desconociendo el carácter arqueológico y su condición de bien patrimonial de la Nación.

Las instituciones públicas y la gestión administrativa son deficiente, porque no aplican procedimientos técnicos, y más bien se basan en empirismos. En ese sentido las gestiones administrativas deben participar todos los organismos de la intuición, para así lograr sinergias efectivas (Rivadeneira, 2020; Silva, 2020).

Los santuarios o bosques, los cuales se encuentran amenazados en la región Lambayeque por parte de migrantes de la región Cajamarca, quienes con autorizaciones por parte de los comuneros en el presente año arrasaron con 25 hectáreas de bosque, lo que llevó a la intervención del ministerio del interior y el ministerio del ambiente, así mismo los disturbios llegaron a lastimar a los integrantes del frente de defensa salvemos a Chaparrí, menciona Mongabay en el portal web (Gamboa, 2016).

Castillo (2014), alega que se encuentra el caso del santuario histórico bosque de Pómac, el cual fue invadido durante 8 años depredando parte del bosque y destruyendo patrimonio cultural de la nación, 
conformado por restos arqueológicos y árboles milenarios, después del posterior desalojo para el año 2009, el bosque comienza con su regeneración.

Dentro de las estrategias de gestión administrativa se tiene a la planificación, en donde se delimita la misión y la visión de la organización, así como su diagnóstico situacional. Otra estrategia es la organización en donde se diseña la estructura orgánica y los documentos de gestión como manuales y reglamentos. Así mismo se tiene la estrategia de dirección en donde se define el estilo de liderazgo seguir y que estrategias de motivación se desarrollaran. Finalmente se tienen control, en donde se hace una retroalimentación (Gamboa, 2016).

Sobre lo mencionado el objetivo de esta investigación fue identificar el nivel de percepción del estado situacional de la gestión administrativa para efectivizar la defensa del patrimonio cultural arqueológico en la región Lambayeque, 2020.

\section{MATERIALES Y MÉTODOS}

El estudio se realizó en el mes de octubre del 2020, mediante la aplicación de encuestas. Los instrumentos fueron validados por tres expertos conocedores de las variables, los cuales estuvieron de acuerdo con la composición de las interrogantes, y consideraron relevantes. La investigación tuvo un enfoque cuantitativo, además es de corte transversal ya que se analizaron los datos y resultados en un solo momento, así mismo el estudio tiene un diseño no experimental, ya que las estrategias de gestión quedaron a modo de propuesta, no se realizó ninguna alteración en las variables para ver sus posibles cambios futuros (Hernández, Fernández y Baptista, 2014).

La población estuvo conformada por los trabajadores del ministerio de cultura que laboran en la región Lambayeque: (01) el encargado de la defensa del patrimonio cultural de la nación en Lambayeque, treinta y tres profesionales en arqueología (33), cincuenta (50) trabajadores de los museos de Lambayeque, diez (10) empleados de la unidad ejecutora Naylamp adscritos al ministerio de cultura y un director como representante del ministerio de cultura en Lambayeque, siendo la muestra 95 trabajadores. Las variables evaluadas fueron la gestión administrativa y el valor del patrimonio cultural.

\section{RESULTADOS}

Tabla 1, se muestra que el $87 \%$ de los encuestados manifiestan la existencia de una inadecuada estrategia de coordinación establecida entre el Ministerio de Cultura con la Policía Nacional del Perú para realizar una eficiente gestión para la defensa del patrimonio arqueológico. 
Tabla 1. Evaluación del nivel de coordinación entre el Ministerio de Cultura y la Policía Nacional del Perú relacionado a la defensa del Patrimonio Arqueológico

\begin{tabular}{lcc}
\hline Escala de medición & Frec. & \% \\
\hline Inadecuado & 66 & $86.8 \%$ \\
Adecuado & 10 & $13.2 \%$ \\
\hline Total & & $100 \%$
\end{tabular}

Tabla 2, se muestra que el 88\% de los encuestados manifiestan la existencia de una inadecuada estrategia de coordinación establecida entre el Ministerio de Cultura con la Fiscalía, con respecto a las denuncias realizadas por delitos que atentan contra el patrimonio cultural, debido a falta de interés por desarrollar estrategias que integren la concientización a la población acerca de identidad cultural.

Tabla 2. Percepción de la coordinación que tiene el Ministerio de Cultura con la Fiscalía ante las denuncias por los delitos en contra del Patrimonio Arqueológicos

\begin{tabular}{lcc}
\hline Escala de medición & Frec. & \% \\
\hline Inadecuado & 67 & $88.2 \%$ \\
Adecuado & 9 & $11.8 \%$ \\
\hline Total & & \\
\hline
\end{tabular}

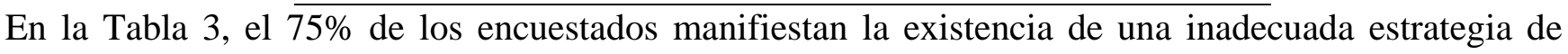
coordinación realizada entre el Ministerio de Cultura y la Defensoría del Pueblo, para promover en las comunidades la importancia de promocionar, proteger y preservar los vestigios culturales, en aras de reconocer su valor irremplazable e inclusión en la toma de decisiones.

Tabla 3. Evaluación de la coordinación para la defensa del Patrimonio Arqueológico entre el Ministerio de Cultura y la Defensoría del Pueblo

\begin{tabular}{lcc}
\hline Escala de medición & Frec. & $\mathbf{\%}$ \\
\hline Inadecuado & 57 & $75 \%$ \\
Adecuado & 19 & $25 \%$ \\
\hline Total & & $100 \%$ \\
\hline
\end{tabular}


En la Tabla 4, el 65\% de los encuestados manifiestan la existencia de estrategias de gestión, entre ellas se tiene adecuada planificación de los recursos que dispone la institución, una correcta organización mediante documentos de gestión, estilo de liderazgo definido y mecanismos de control. Las estrategias que se recomiendan aplicar para una eficiente defensa del patrimonio cultural son: Tener una visión y misión definida y socializada entre los organismos encargados de la defensa del patrimonio, así mismo la organización debe contar con un manual de organización y funciones, así como un reglamento de defensa patrimonial. Finalmente, otra estrategia que se recomienda es aplicar un liderazgo transformacional y establecer estándares de medición.

Tabla 4. Estrategias de gestión para efectivizar la defensa del patrimonio cultural arqueológico en la región Lambayeque

\begin{tabular}{|c|c|c|c|c|c|}
\hline Estrategias & $\begin{array}{c}\text { Institución Museos } \\
\text { Patrimonio Arqueológico }\end{array}$ & Frecuencia & $\%$ & Inadecuado & Adecuado \\
\hline Planificación & $\begin{array}{l}\text { Visión y misión definida y } \\
\text { socializada entre los } \\
\text { organismos encargados de la } \\
\text { defensa del patrimonio. }\end{array}$ & 10 & 13 & 10 & 0 \\
\hline Organización & $\begin{array}{llr}\text { Contar con } & \text { un manual de } \\
\text { organización y funciones y } \\
\text { reglamento } \\
\text { patrimonial. }\end{array}$ & 17 & 22 & 17 & 0 \\
\hline Dirección & $\begin{array}{ll}\text { Aplicar un } & \text { liderazgo } \\
\text { transformacional } & \\
\end{array}$ & 20 & 26 & 0 & 20 \\
\hline \multirow[t]{2}{*}{ Control } & $\begin{array}{lll}\begin{array}{l}\text { Establecer } \\
\text { medición. }\end{array} & \text { estándares } & \text { de } \\
\end{array}$ & 29 & 38 & 0 & 29 \\
\hline & Total & 76 & 100 & 27 & 49 \\
\hline
\end{tabular}

\section{DISCUSIÓN}

El $87 \%$ de los encuestados manifiestan la existencia de una inadecuada estrategia de coordinación establecida entre el Ministerio de Cultura con la Policía Nacional del Perú para realizar una eficiente gestión en la defensa del patrimonio arqueológico. Los hallazgos se relacionan con Logacho (2019), en donde manifiesta que sus áreas de influencia ceremonial, presentan una infraestructura en alto grado de deterioro, ocasionado por la falta de inversión pública y la escaza coordinación entre entidades públicas encargadas de velar por el cuidado del patrimonio nacional. Para Elbanna y Andrews (2016) debe existir un liderazgo definido en las instituciones públicas con la finalidad que se puedan conducir de manera 
adecuada los procesos de defensa del patrimonio cultural, pues según los resultados se tiene niveles altos de un 87\% de una inadecuada gestión. De igual forma Melgar y Antón (2019), manifiestan que se debe involucrar a la sociedad civil para que contribuyan con la defensa patrimonial, entre estas instituciones deben estar las universidades, entidades privadas como bancos, supermercados, así mismo instituciones educativas de nivel básico y colegios profesionales.

Como se puede evidenciar estas investigaciones manifiestan que los patrimonios culturales deben ser valorizados y potenciados, pues según los hallazgos presentan indicadores altos con un 87\% donde se alega que el ministerio de cultura no está realizando una adecuada potenciación del patrimonio cultural. Pérez (2015) la puesta en valor del paisaje arqueológico cultural de Coyoctor, indica que el propósito es potencializar el patrimonio histórico cultural y promover la gestión creativa de sus recursos turísticos. También Cevallos (2019) menciona que el diseño arquitectónico del centro cultural y museo de sitio no se está exponiendo al mundo, y esto en gran medida por que los accesos y vías de comunicación no son las adecuadas. Por lo que se requiere el apoyo de las instituciones públicas y privadas. Los resultados encontrados se sustentan en la teoría de Rodríguez (2015), el cual indica que el patrimonio cultural la herencia cultural propia del pasado de una comunidad, mantenida hasta la actualidad y transmitida a las generaciones.

El 88\% de los encuestados manifiestan la existencia de una inadecuada estrategia de coordinación establecida entre el Ministerio de Cultura con la Fiscalía, con respecto a las denuncias realizadas por delitos que atentan contra el patrimonio cultural, debido a falta de interés por desarrollar estrategias que integren la concientización a la población acerca de identidad cultural. Los hallazgos se asemejan a la investigación de Loaiza (2016) en su artículo sobre modelo de gestión administrativa, manifiesta que las instituciones públicas deben tener un esquema de planificación que permita tener una visión y crecimiento organizacional. Laime (2017) indica que las instituciones públicas deben tener diferentes procesos y controles para cumplir con los objetivos.

El 75\% de los encuestados manifiestan la existencia de una inadecuada estrategia de coordinación realizada entre el Ministerio de Cultura y la Defensoría del Pueblo, para promover en las comunidades la importancia de promocionar, proteger y preservar los vestigios culturales, en aras de reconocer su valor irremplazable e inclusión en la toma de decisiones.

Rodríguez (2019), referente a gestión administrativa del servicio público, indica que se debe planificar, organizar dirigir y controlar adecuadamente los recursos de las instituciones, para así poder lograr los objetivos de manera efectiva. Es necesario establecer indicadores de eco eficiencia para poder tener un 
impacto de la gestión. Para Ochoa y Zogheib (2019) se debe tomar decisiones pertinentes que sean significativas en la gestión administrativa. Las instituciones deben contar con un sistema de planificación de defensa del patrimonio, apoyado en una organización eficiente y un liderazgo efectivo. Según los resultados, no se está coordinando adecuadamente entre instituciones encargadas de velar por el patrimonio cultural.

\section{CONCLUSIONES}

Las estrategias de gestión administrativa que permiten efectivizar la defensa del patrimonio cultural, son las acciones adecuadas en planificación de los recursos que dispone la institución, así mismo una correcta organización mediante documentos de gestión. Igualmente, un estilo de liderazgo definido y finalmente establecer mecanismos de control. Sin embargo, se evidencia poca cooperación por parte de los organismos implicados en la defensa del patrimonio cultural, así como la falta de presupuesto y una mala distribución del mismo.

La actual gerencia viene desarrollando de manera inadecuada la gestión administrativa, pues se evidencia que los indicadores de gestión están por debajo de lo esperado, mostrando ineficiencia en cuanto a la defensa del patrimonio cultural arqueológico de la región Lambayeque, pues no se tiene un adecuado registro, declaración, protección, restauración, educación y sanción; del patrimonio arqueológico.

\section{AGRADECIMIENTOS}

A la Universidad Privada Cesar Vallejo de la filial de Chiclayo porque brinda la oportunidad de poder compartir las investigaciones con la comunidad científica.

\section{REFERENCIAS BIBLIOGRÁFICAS}

Alva, W. (02 de Diciembre de 2016). Lambayeque: dnuncian invasión de la zona arqueológica de Mata Indio. Obtenido de Andina: http://www.andina.com.pe/agencia/noticia-lambayeque-denuncianinvasion-de-zona-arqueologica-mata-indio-587627.aspx

Baeza, V. (05 de Enero de 2015). La otra huella del Dakar: “El daño a sitios arqueológicos es irreparable”. Obtenido de Explora: http:/www.explora.cl/428-articulos-de-ciencia/articulos-otrashumanidades-y-ciencias-sociales-1/4628-la-otra-huella-del-dakar-el-dano-a-sitiosarqueologicos-es-irreparable 
Castillo, A.; Ménendez, S. (2014). Managing urban archaeological heritage: Latin American case studies. International Journal of Cultural Property, 21: 55-77.

Cevallos Jaramillo, E. (2019). Diseño arquitectónico del centro cultural y museo de sitio del “Complejo y Parque Arqueológico Cochasquí”, para el cantón Pedro Moncayo, provincia de Pichincha en base a un entendimiento infraestructural del proyecto. Trabajo de titulación previo a la obtención del Título de Arquitecto. Carrera de Arquitectura. Quito: UCE. 134 p.

Elbanna, D \& Fadol, Y (2016) The Role of Context in Intuitive Decision Making. Cambridge University. Gamboa, J (2016) Patrimonio Arqueológico en riesgo: conservación, destrucción y perspectivas de conservación de sitios precolombinos en Trujillo, Perú https://revistas.ucm.es/index.php/CMPL/article/view/54748

Hernández, R.; Fernández, C.; y Baptista, P. (2014). Metodología de la investigación. México D.F., México: McGraw Hill Education.

Laime Guamán, Karina Elizabeth (2017). Diseño de un Sistema de Gestión Administrativo - Financiero de la empresa VERITEX ubicada en la Ciudad de Quito. Trabajo de Titulación, modalidad proyecto de investigación para la obtención del Título de Ingeniera en Contabilidad y Auditoría. Contadora Pública Autorizada. Carrera de Contabilidad y Auditoría. Quito: UCE. 101 p.

Loaiza Hurtado, Katherine Johanna (2016). Propuesta de un modelo de gestión administrativa y financiera para la empresa Intiwasi, en el Distrito Metropolitano de Quito. Trabajo de investigación para optar por el Título de Ingeniera en Contabilidad y Auditoría. Contadora Pública Autorizada. Carrera de Contabilidad y Auditoría. Quito: UCE. 113 p.

Logacho Simbaña, T. y Morales Quinga, K. (2019). El Patrimonio Arqueológico InkaWasi y sus áreas de influencia ceremonial en la parroquia Caranqui, cantón Ibarra. Trabajo de titulación previo a la obtención del Título de Licenciado en Ciencias de la Educación. Mención Ciencias Sociales. Carrera de Ciencias Sociales. Quito: UCE. 209 p.

Melgar, A, Flores, W, Arevalo, J y Anton, P (2019) Tecnologías educativas, habilidades sociales y la toma de decisiones. Artículo científico. ScieloPeru.

Noreña, S (2013) Divulgación, difusión y valoración del patrimonio arqueológico. Boletín de Antropología

Ochoa, B y Zogheib, Ch (2019) ¿Estamos tomando decisiones basadas en evidencia para la gestión del agua?. Revista SPDA Actualidad Ambiental. 
Pérez Bermeo, Nohemí Verónica (2015). Interpretación y puesta en valor del paisaje arqueológico cultural de Coyoctor en la provincia de Cañar. Trabajo de grado previo a la obtención del título de Licenciada en Turismo Histórico y Cultural. Carrera de Turismo Histórico. Quito: UCE. 120 p.

Rivadeneira, A (2020) Seguimiento y evaluación de los planes estratégicos en el sector salud: estudio de caso, año 2018. Tesis doctoral.

Rodríguez Loor, B. (2019). La gestión administrativa del servicio público en el Distrito de Educación 13d07 Chone-Flavio Alfaro, Período (2015- 2017). Trabajo de titulación previo a la obtención del Título de Magister en Gestión Pública. Instituto de Investigación y Posgrado. Quito: UCE. $121 \mathrm{p}$.

Rodriguez, F. (2015). Un entendimiento infraestructural del proyecto arquitectónico. Madrid: Escuela Técnica Superior de Arquitectura de Madrid

Silva, M (2020) Plan estratégico multisectorial para la reducción de la contaminación acústica por ruido vehicular en la ciudad de Chachapoyas. Tesis doctoral. Universidad Cesar Vallejo. 